\title{
Parameters of hydrothermal gelation of chitin nanofibers determined using a severity factor
}

Shin Suenaga and Mitsumasa Osada*

Department of Chemistry and Materials, Faculty of Textile Science and Technology, Shinshu University, 3-15-1, Tokida, Ueda, Nagano 386-8567, Japan

*Corresponding Author

E-mail: osadam@ shinshu-u.ac.jp; Tel.: +81-268-21-5458; Fax: +81-268-21-5391 


\section{Abstract}

The hydrothermal gelation of $\alpha$ - and $\beta$-chitin nanofibers ( $\alpha$ - and $\beta$-ChNFs) prepared at neutral and acidic $\mathrm{pH}$ was conducted by heating them to $120,160,180$, and $200{ }^{\circ} \mathrm{C}$ in a sealed reactor. The optical transmittance and mechanical strength of $\beta$-ChNFs gelated at the acidic $\mathrm{pH}$ were determined for the first time using a severity factor defined as a function of the integrated heating time and temperature. The width of $\beta$-ChNFs increased after the hydrothermal treatment, indicating that these fibers strongly adhered to each other to form a network structure during gelation. Furthermore, the hydrothermal gelation of $\alpha$ - and $\beta$-ChNFs with different degrees of disintegration prepared at the neutral and acidic $\mathrm{pH}$ was conducted. It was found that the hydrothermal treatment of $\alpha$-chitin must be performed at the acidic $\mathrm{pH}$ to obtain a self-sustaining hydrogel of well-disintegrated NFs. The disintegration of $\beta$-chitin into NFs occurred more easily at the acidic $\mathrm{pH}$ than under the neutral conditions; however, in the latter case, the same disintegration degree of $\beta$-ChNFs could be achieved by increasing the number of disintegration steps. At the same disintegration degree, the strength of the self-sustaining hydrogel obtained at the neutral conditions was greater than that of the gel prepared at the acidic $\mathrm{pH}$, indicating that the electrostatic repulsion caused by acid addition negatively affected the formation of the hydrogel network structure. To maximize the efficiency of the hydrothermal gelation process, ChNFs should be as thin as possible and electrostatic repulsion forces must be controlled.

Keywords: $\alpha$-chitin nanofiber, $\beta$-chitin nanofiber, hydrothermal gelation, severity factor 


\section{Introduction}

Chitin is an abundant biomass resource generated in the amount of about 100 billion tons per year by organisms (Yan and Chen 2015). It is widely utilized in medical supplies (Sowmya et al. 2011; Izumi et al. 2015), cosmetics (Gautier et al. 2008), and food (Han et al. 1999) because its acetamide group located at the $C 2$ position strongly affects the biochemical properties of chitin (Jayakumar et al. 2009; Madhumathi et al. 2009). Chitin has been studied in various forms, including the hydrogel one (Tamura et al. 2006; Mushi et al. 2016), which can be prepared by chemical cross-linking (Chang et al. 2011), immersion into alkaline solution (Abe et al. 2014), and recrystallization from solution (Shen et al. 2016). However, these methods also have the following disadvantages: (1) a complicated organic synthesis procedure due to the existence of multiple steps; (2) environmental contamination caused by organic, acidic, and alkaline waste liquids; and (3) high cytotoxicity of the utilized chemical reagents.

Recently, hydrothermal gelation has attracted much attention as a new gelation method that does not require additives (Nata et al. 2012; Lewis et al. 2016; Suenaga and Osada 2018a). Nata et al. have reported a successful conversion of a dispersion of $\beta$-chitin nanofibers $(\beta$ ChNFs) into a self-sustaining hydrogel inside an autoclave at a temperature of $180{ }^{\circ} \mathrm{C}$ (Nata et al. 2012). This method is relatively simple because it only involves heating NF dispersion in a sealed reactor and produces minimal negative effect on the environment because of reduction of the input energy required for the evaporation of water. In addition, the hydrogel produced by hydrothermal treatment is expected to preserve the high biocompatibility of chitin after gelation because of the absence of any additives. Although the hydrothermal gelation process has many advantages, further studies must be conducted to determine its optimal conditions. In a previous work, it was reported that gelation did not occur at temperatures below $180{ }^{\circ} \mathrm{C}$ (Nata et al. 2012). On the other hand, it can be assumed that the gelation process likely occurs after either a prolonged heating at a lower temperature or shorter heating at a higher temperature. Thus, the optimal heating time is directly related to temperature, which can be described by the severity factor $R_{0}$ defined as a function of the integrated heating time and temperature and expressed by the following equation (Klinchongkon et al. 2015):

$$
\log R_{0}=\log \left\{\int_{0}^{t} \exp \left(\frac{T(t)-100}{14.75}\right) \mathrm{d} t\right\}
$$

where $t$ is the heating time [s], and $T$ is the heating temperature $\left[{ }^{\circ} \mathrm{C}\right]$. This parameter has been proposed by Overend and Chornet in a study describing the production of Kraft pulp from trees using sub-critical and super-critical water treatment procedures (Overend and Chornet 1987). The pulps prepared at the same values of $R_{0}$ and different magnitudes of the heating temperature and time exhibited similar quality.

In this study, the hydrothermal treatment of ChNF dispersions was performed at temperatures of $120,160,180$, and $200{ }^{\circ} \mathrm{C}$ and the same values of $\log R_{0}$, and the physicochemical properties of the resulting hydrogels were examined. Previously, the hydrothermal gelation of chitin fibers was conducted for only $\beta$-ChNFs at acidic $\mathrm{pH}$ (Nata et al. 2012). However, we have also found that the physicochemical properties of $\alpha$-chitin nanofibers ( $\alpha$-ChNFs) and $\beta$-ChNFs strongly depended on their crystalline structure, acidity, and disintegration degrees (Suenaga et al. 2017; Suenaga and Osada 2018b). Hence, the objective of this study was to evaluate the possibility of transforming various types of ChNFs into self-sustaining hydrogels via hydrothermal treatment and determine the most important parameters of the hydrothermal gelation of $\alpha$ - and $\beta$-ChNFs with different disintegrating properties prepared at the neutral and acidic conditions. 


\section{Materials and methods}

\section{Materials}

$\alpha$-Chitin with particle sizes below $100 \mu \mathrm{m}$ was obtained from Yaegaki Bio-industry, Inc. (Himeji, Japan) and used without further purification. $\beta$-Chitin was purified from squid pens (Todarodes pacificus) after removing ash and protein via its treatment with hydrochloric acid and sodium hydroxide according to our method developed in a previous work (Suenaga et al. 2016). The purified $\beta$-chitin was pulverized to particles with sizes of approximately $100 \mu \mathrm{m}$ using a dry pulverizer (Cyclone Mill, Nippon Steel \& Sumikin Fine Technology Co., Ltd., Osaka, Japan). Hydrochloric acid, sodium hydroxide, and acetic acid were purchased from Wako Pure Chemical Industries (Osaka, Japan) and used without further purification.

\section{Preparation of ChNFs}

The $\alpha$ - and $\beta$-chitin powders were separately suspended in distilled water at a concentration of $1 \mathrm{w} / \mathrm{v} \%$. The measured $\mathrm{pH}$ of the prepared chitin slurries was $6.5-7.5$, so the $\mathrm{pH}$ is expressed as 7 in this work. These slurries were disintegrated into NFs using a Star Burst system (Star Burst Mini, Sugino Machine Co., Ltd., Uozu, Japan) equipped with a ball-collision chamber. To obtain ChNFs with $\mathrm{pH}=4$, dilute acetic acid was added to the prepared chitin slurries followed by their disintegration using a Star Burst system. In the disintegration process, the slurries pressurized at approximately $235 \mathrm{MPa}$ were ejected from the nozzle with an aperture size of $100 \mu \mathrm{m}$ and collided with a ceramic ball. The disintegrated samples were cooled at a temperature of $25^{\circ} \mathrm{C}$ using a heat exchanger and then recovered. The number of collisions with the ceramic ball (passes) was set to either 10 or 30 . The $\mathrm{pH}$ of the dispersion was measured before and after disintegration using a KR5E pH meter equipped with an LE407 module (As One Co., Ltd., Osaka, Japan). The $\alpha$ - and $\beta$-ChNFs were heated to $40{ }^{\circ} \mathrm{C}$ for $10 \mathrm{~min}$ in a thermostat chamber filled with tap water, stirred at a speed of $2000 \mathrm{rpm}$ for $1 \mathrm{~min}$, and then defoamed at a speed of $2000 \mathrm{rpm}$ for $1 \mathrm{~min}$ using an ARE-250 mixer (Thinky Co., Ltd., Tokyo, Japan) to prevent the formation of bubbles in the hydrogels after the hydrothermal treatment.

\section{Gelation via hydrothermal treatment}

Approximately $3 \mathrm{~mL}$ of $\alpha$ - or $\beta$-ChNFs was loaded into a stainless steel 316 tube reactor (volume: $6 \mathrm{~cm}^{3}$ ), which was subsequently immersed into a molten-salt $\left(\mathrm{KNO}_{3}-\mathrm{NaNO}_{3}\right)$ bath with a temperature of 160,180 , or $200{ }^{\circ} \mathrm{C}$. For the hydrothermal treatment at $120{ }^{\circ} \mathrm{C}$, an oil bath was used. The saturated vapor pressures in the reactor calculated at 120, 160, 180, and $200{ }^{\circ} \mathrm{C}$ were equal to $0.20,0.62,1.00$, and $1.55 \mathrm{MPa}$, respectively (Wagner and Pruß 2002). The temperature inside the reactor was directly measured by inserting a thermocouple into it. The heating time was set to $2 \mathrm{~d}$ and $10 \mathrm{~h}$ at $120^{\circ} \mathrm{C} ; 7,15,30,45,60,120,240,480,720$, and $960 \mathrm{~min}$ at $160{ }^{\circ} \mathrm{C} ; 1.5,3,7,15,30,60,120,180$, and $240 \mathrm{~min}$ at $180^{\circ} \mathrm{C}$; and $1.5,3,6,12,15$, 30,45 , and $60 \mathrm{~min}$ at $200{ }^{\circ} \mathrm{C}$ (their magnitudes included the heat-up time, as shown in Fig. 1). After the heating process, the reactor was removed from the molten-salt or oil bath and rapidly quenched in a water bath to $25^{\circ} \mathrm{C}$. The produced hydrogel was recovered from the reactor on a glass dish. 


\section{Optical transmittance measurements}

The prepared hydrogels were ground with a pestle and mortar to obtain flowing pastes. Each paste was loaded into a quartz cuvette, and its transmittance was measured in the range of 200-700 nm at $25^{\circ} \mathrm{C}$ using a spectrophotometer (V530, Jasco Co., Ltd., Tokyo, Japan) equipped with a Peltier-type temperature controller (ETC-505, Jasco Co., Ltd.) and distilled water as the blank. The transmittance at a wavelength of $600 \mathrm{~nm}$ and the wavelength at a transmittance of less than $0.1 \%$ were determined. The former parameter was used as a representative value for the visible light, and the latter one indicated the degree of the brown discoloration of the hydrogel observed after the hydrothermal treatment.

\section{Compression testing}

The prepared hydrogel samples were cut into pieces with diameters of $8 \mathrm{~mm}$ and heights of approximately $5 \mathrm{~mm}$ using a razor. Compression tests were performed using an EZ Test machine (Shimadzu, Co., Ltd., Tokyo, Japan) with a $50 \mathrm{~N}$ load cell at a compression rate of 1 $\mathrm{mm} \mathrm{min}^{-1}$ and room temperature. As a result, the values of the breaking strength and compressive modulus at $4-8 \%$ of the initial strain were determined.

\section{Field-emission scanning electron microscopy observations}

The hydrogel samples were cut into pieces with diameters of $8 \mathrm{~mm}$ and heights of approximately $2 \mathrm{~mm}$. Subsequently, both ChNFs and the cut specimens were immersed in tertbutyl alcohol for $3 \mathrm{~d}$. After the treatment with tert-butyl alcohol more than 5 times, the ChNFs and specimens were quickly frozen and dried under vacuum (0.02 MPa) (MDA-015, ULVAC KIKO Inc., Miyazaki, Japan) for a few hours. The dried samples were coated with a $2.5 \mathrm{~nm}$ thick layer of osmium using an osmium coater (Neoc-STP, Meiwafosis Co., Ltd., Tokyo, Japan). The coated specimens were observed by a field-emission scanning electron microscope (FESEM; S-5000, HITACHI Co., Ltd., Tokyo, Japan) operated at a voltage of $5.0 \mathrm{kV}$. A histogram of ChNF widths was constructed from four different FE-SEM images ( $80 \mathrm{ChNF}$ widths were measured during analysis) using the Image-J software (NIH, Bethesda, USA). The average width of ChNFs was calculated as number average width.

\section{Fourier-transform infrared spectroscopy studies}

ChNFs or the hydrogel samples were frozen at $-80{ }^{\circ} \mathrm{C}$ inside a freezer and then lyophilized for $1 \mathrm{~d}$ using a freeze dryer (FDU-2200, Tokyo Rikakikai Co., Ltd., Tokyo, Japan). The lyophilized samples were washed with about $400 \mathrm{~mL}$ of distilled water to remove the remaining traces of acetic acid, after which the lyophilization procedure was repeated again. The dried samples were blended with $\mathrm{KBr}$ and then pressed into pellets using a mini hand-press (Shimadzu Co., Ltd.). Fourier-transform infrared (FT-IR) spectra were recorded by performing 32 scans in the range of $500-4000 \mathrm{~cm}^{-1}$ at a resolution of $4 \mathrm{~cm}^{-1}$ using an FT/IR 4200 spectrometer (Jasco Co., Ltd.). The degree of $N$-acetylation was calculated via the following equation (Brugnerotto et al. 2001):

$$
\text { Degree of } N \text {-acetylation }=\frac{A 1320 / A 1420-0.3822}{0.03133}
$$


where $A 1320$ and $A 1420$ are the absorbance values measured at 1320 and $1420 \mathrm{~cm}^{-1}$, which correspond to the stretching and bending vibrations of amide III and $\mathrm{CH}_{2}$ groups, respectively. Their magnitudes were determined via the baseline method proposed in a previous study (Brugnerotto et al. 2001).

\section{X-ray diffraction analysis}

The dried samples were converted into pellets (diameter: $1.3 \mathrm{~cm}$, thickness: $0.3 \mathrm{~mm}$ ) by pressing at a pressure of $750 \mathrm{MPa}$ for 1 min using a hand-press (SSP-10A, Shimadzu Co., Ltd.). XRD patterns were recorded in the $2 \theta$ range of $5-35^{\circ}$ using an X-ray diffractometer (RINT $2500 \mathrm{HF} / \mathrm{PC}$, Rigaku Co., Ltd.) with $\mathrm{Cu}-K \alpha$ radiation $(\lambda=1.5418 \AA$ ) operated at a voltage of 40 $\mathrm{kV}$ and current of $40 \mathrm{~mA}$. The crystallinity index (CI) was determined using the following equation (Zhang et al. 2005):

$$
\mathrm{CI}=\left(I_{1-10}-I_{\mathrm{am}}\right) / I_{1-10}
$$

where $I_{1-10}$ is the peak intensity of the [1-10] plane, and $I_{\mathrm{am}}$ is the intensity of the amorphous diffraction at $16^{\circ}$. The $d$-spacing value was obtained using Bragg's law:

$$
d \text {-spacing }[\AA]=\lambda / 2 \sin \theta
$$

where $\lambda$ is the wavelength of $\mathrm{Cu}-K \alpha$ radiation, and $\theta$ is the scattering angle. The crystal size (CS) was determined using the Scherrer equation:

$$
\mathrm{CS}[\mathrm{nm}]=k \lambda / \beta_{0} \cos \theta
$$

where $k$ is the constant describing the shape factor (0.9), and $\beta_{0}$ is the full width at halfmaximum of the diffraction peak.

\section{Results and discussion}

\section{Physicochemical properties of $\beta$-ChNF hydrogels prepared at $\mathrm{pH}=4$ and 30 passes}

Temperature profiles inside the reactor and calculation method of the severity factor

The temperature profiles inside the reactor obtained for different heating temperatures and times are displayed in Fig. 1. They show that the heating time decreased with increasing target temperature (the curve bending observed during the heat-up stage resulted from the remelting of the salt coagulated around the reactor). The values of $\log R_{0}$ during heat-up were calculated via the trapezoidal integration of the temperature function using a grid with a spacing of $0.2 \mathrm{~s}$. 


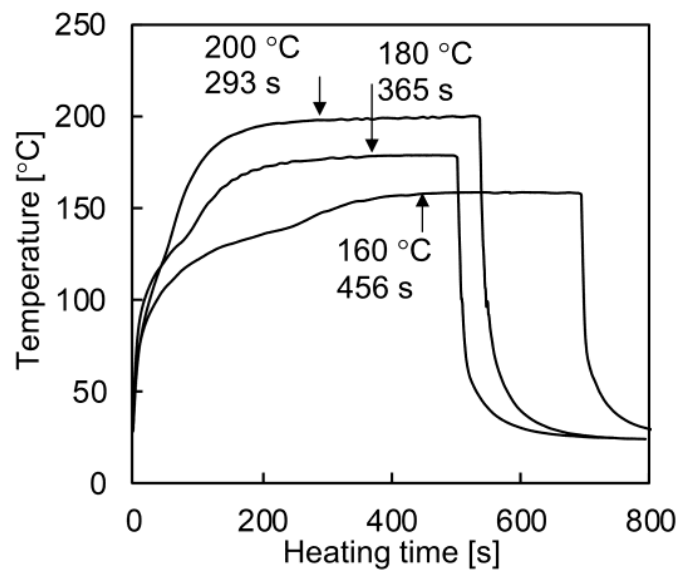

Fig. 1 Temperature profiles inside the reactor obtained for different heating temperatures. The arrows indicate the points where the target temperatures were reached

Effect of the severity factor on the physicochemical properties of the hydrogel

First, the similarity of the physicochemical properties of the self-sustaining hydrogels with the same severity factors $\left(\log R_{0}\right)$ observed at different heating temperatures was confirmed by testing the $\beta$-ChNFs samples prepared at $\mathrm{pH}=4$ and subjected to 30 passes under the conditions identical to those utilized in a previous study (Nata et al. 2012).

The transmittance at a wavelength of $600 \mathrm{~nm}$ and wavelength at a transmittance of below $0.1 \%$ plotted as functions of $\log R_{0}$ and the heating time are shown in Figs. 2 and $\mathrm{S} 1$, respectively (the ultraviolet-visible spectra of the hydrogels recorded in the range of 200-700 $\mathrm{nm}$ are shown in Fig. S2, and their photographs obtained at various conditions are depicted in Fig. S3). They demonstrate the decrease in transmittance and red shift of the wavelength with an increase in $\log R_{0}$ regardless of the heating temperature. As shown in Fig. S1, when the transmittances of the $\beta$-ChNFs samples are plotted against the heating time, they exhibit only decreasing trends at each temperature. However, their dependences on $\log R_{0}$ can be characterized by a single curve. The discoloration of the specimens observed after the hydrothermal treatment (Fig. S3) likely resulted from either caramelization or the Maillard reaction, and the corresponding degree of $\beta$-ChNFs decomposition could be estimated from the magnitude of $\log R_{0}$. 

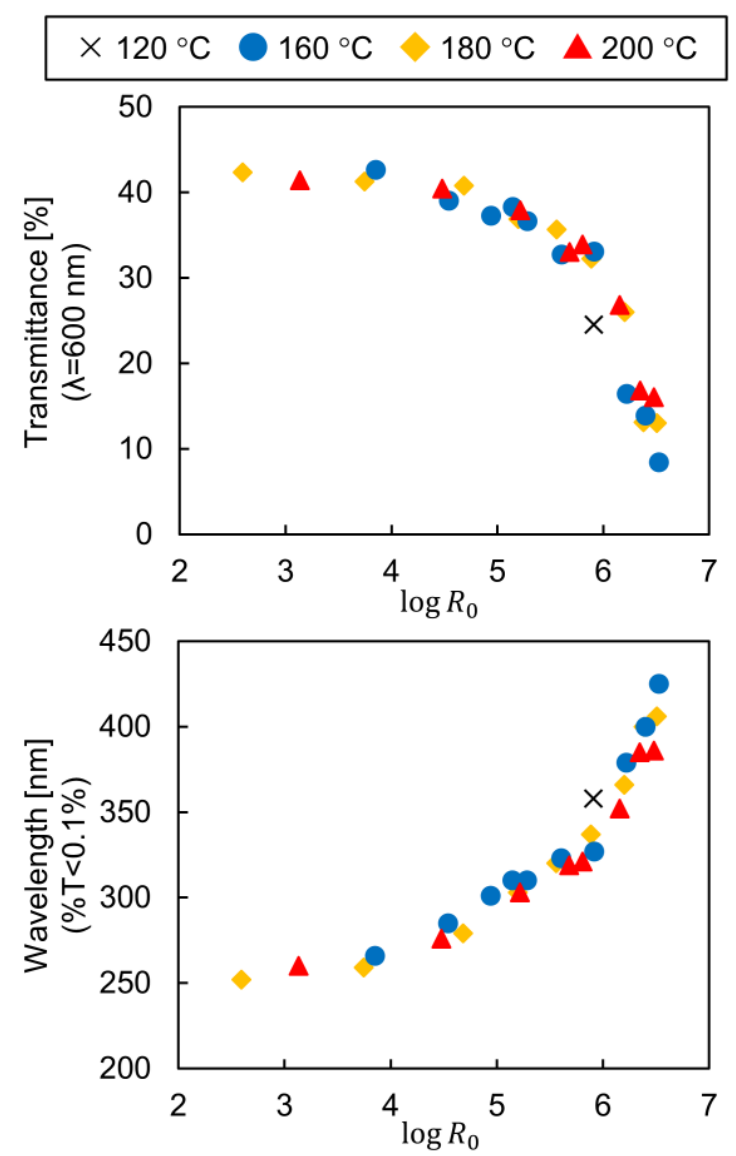

Fig. 2 Optical transmittances of the $\beta$-ChNF hydrogels prepared at $\mathrm{pH}=4$ and 30 passes as functions of the severity factor $\log R_{0}$

The breaking strengths and compressive moduli of the hydrogels prepared from the $\beta$ ChNFs subjected to 30 passes, which are plotted as functions of $\log R_{0}$ and the heating time, are shown in Figs. 3 and S4, respectively. They demonstrated similar trends with increasing log $R_{0}$ regardless of the heating temperature. In particular, the hydrogel breaking strength increased until reaching a magnitude of $\log R_{0} \approx 5.6$ and leveled out at around $1.7 \mathrm{kPa}$ (the compressive modulus also increased with increasing $\log R_{0}$ ). Therefore, similar to transmittance, the mechanical properties of the hydrogels can be estimated by calculating $\log R_{0}$. 

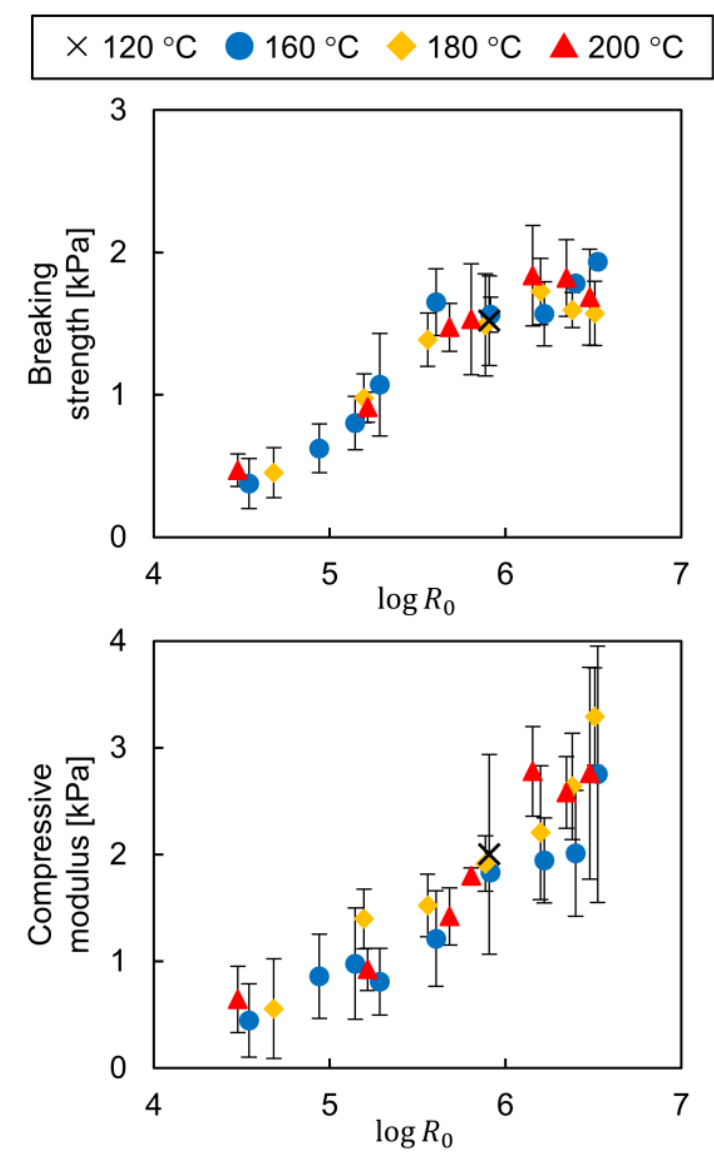

Fig. 3 Mechanical properties of the $\beta$-ChNF hydrogels prepared at $\mathrm{pH}=4$ and 30 passes as functions of the severity factor $\log R_{0}$

Further, the dependences of the gelation reaction parameters on the heating temperature and utilized equipment were investigated. The physicochemical properties of the hydrogel obtained by heating $\beta$-ChNFs to $120^{\circ} \mathrm{C}$ using the oil bath are denoted by the crosses in Figs. 2 and 3 , whose positions are almost identical to those of the other hydrogels with the same $\log R_{0}$ values. These results indicate that the hydrogels with similar properties can be obtained if their corresponding $\log R_{0}$ magnitudes are identical. Hence, the utilization of the $\log R_{0}$ parameter can facilitate the determination of suitable heating conditions during the hydrothermal gelation and thus expands application range of the produced hydrogels.

The width distributions of the original $\beta$-ChNFs prepared at $\mathrm{pH}=4$ and 30 passes and corresponding hydrogels as well as their widths plotted versus the severity factor are shown in Fig. 4, while their typical FE-SEM images are presented in Fig. S5. The obtained results indicate that thin $\beta$-ChNFs are well entangled with each other under all conditions. Furthermore, the average width of the original $\beta$-ChNFs was $10.1 \mathrm{~nm}$, whereas the width of $\beta$-ChNFs in the produced hydrogels increased with increasing $\log R_{0}$ and reached a magnitude that was about 1.5 times greater than that of the original $\beta$-ChNFs at $\log R_{0}>6$, suggesting that two NFs formed a network structure through adherence. 

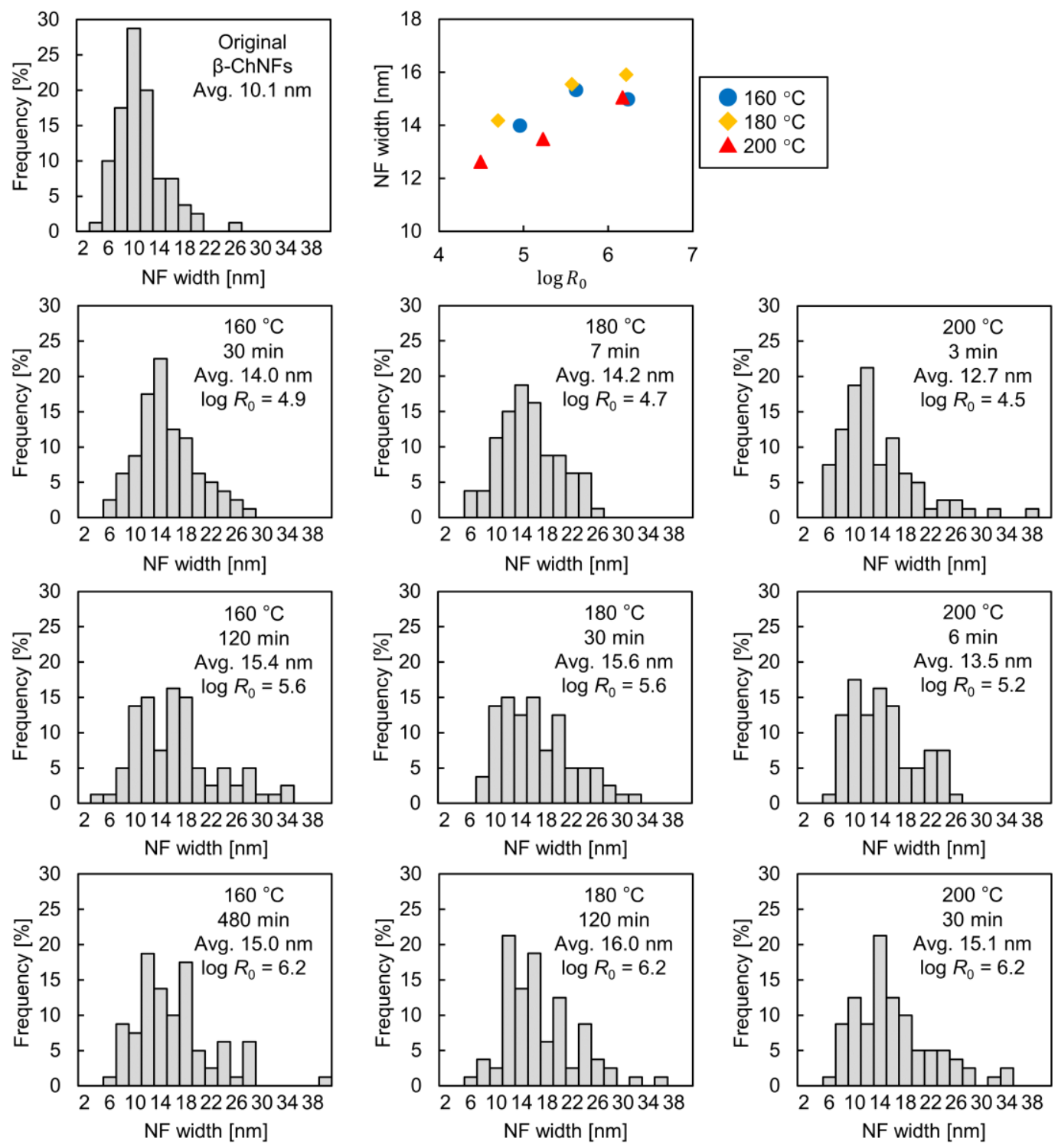

Fig. 4 Histograms characterizing the width distributions of $\beta$-ChNFs before and after the hydrothermal treatment. The upper right panel displays the average width of $\beta$-ChNFs plotted as a function of the severity factor $\log R_{0}$. The values in the histogram panels indicate heating temperature, heating time, average width of the $\beta$-ChNFs, and severity factor

\section{Chemical and crystalline structure of hydrogel before and after hydrothermal gelation}

The FT-IR spectra of the original $\beta$-ChNFs prepared at $\mathrm{pH}=4$ and 30 passes and their corresponding hydrogels as well as the degree of $N$-acetylation plotted as a function of the severity factor are shown in Fig. 5. No significant differences were observed between the $\beta$ $\mathrm{ChNFs}$ and hydrogels at various heating temperatures and times. The degree of $N$-acetylation of the original $\beta$-ChNFs was $95 \%$, and their deacetylation did not occur after the hydrothermal treatment. In previous studies, the decomposition of the chitin chemical structure through its deacetylation was observed after the hydrothermal treatment at temperatures above $300{ }^{\circ} \mathrm{C}$ (Osada et al. 2012, 2013, 2015; Aida et al. 2014); however, the hydrothermal treatment at temperatures less than $200{ }^{\circ} \mathrm{C}$ conducted in this study did not decrease the degree of $\mathrm{N}$ acetylation. The obtained FT-IR spectra indicate that the chemical structure of $\beta$-ChNFs was not affected by the hydrothermal gelation. 


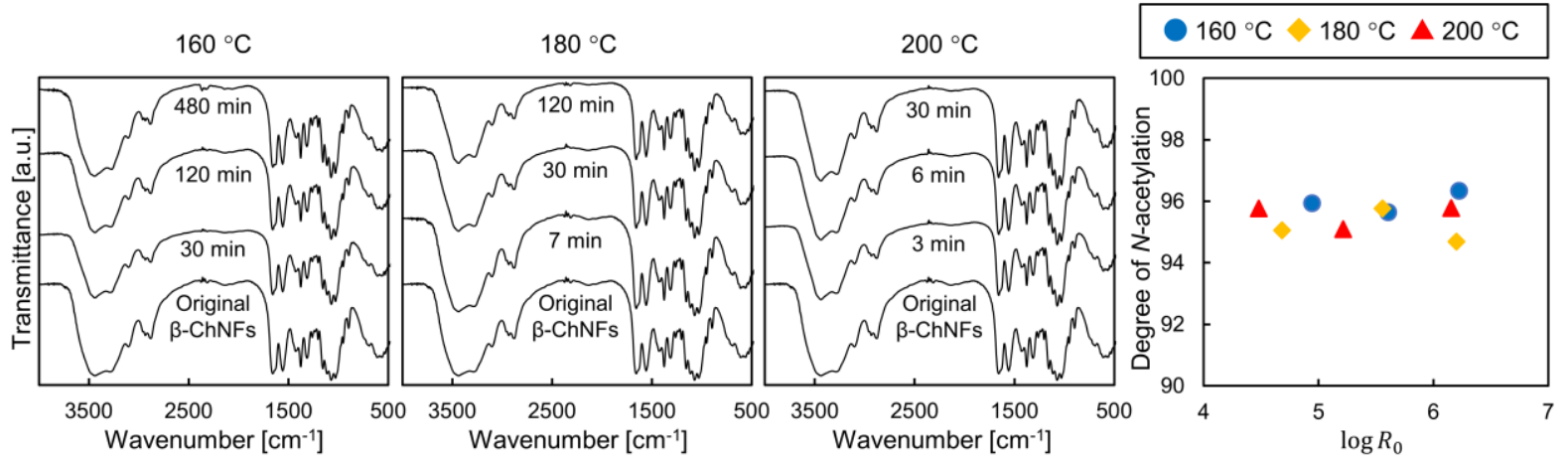

Fig. 5 FT-IR spectra of $\beta$-ChNFs recorded before and after the hydrothermal treatment and their degrees of $N$ acetylation plotted as functions of the severity factor $\log R_{0}$

The XRD patterns of the original $\beta$-ChNFs prepared at $\mathrm{pH}=4$ and 30 passes and corresponding hydrogels are shown in Fig. 6, and their CI, $d$-spacing, and CS values are listed in Table 1. The patterns obtained for the hydrogel samples were identical to those of the original $\beta$-ChNFs regardless of $\log R_{0}$, indicating that the gelation via hydrothermal treatment occurred due to the interactions between individual $\beta$-ChNFs (which preserved their crystalline structure) rather than through the dissolution followed by reprecipitation (in the latter case, the crystal structure would be transformed into an $\alpha$-type one). The detailed calculations performed for the XRD patterns showed that the CI and CS values obtained for the [1-10] mixed plane increased after the hydrothermal treatment, while the magnitude of $d$-spacing remained unchanged. According to the results of a previous study, annealing to high temperatures caused the rearrangement of the NF crystal structure (Nata et al. 2012; Suenaga et al. 2017).
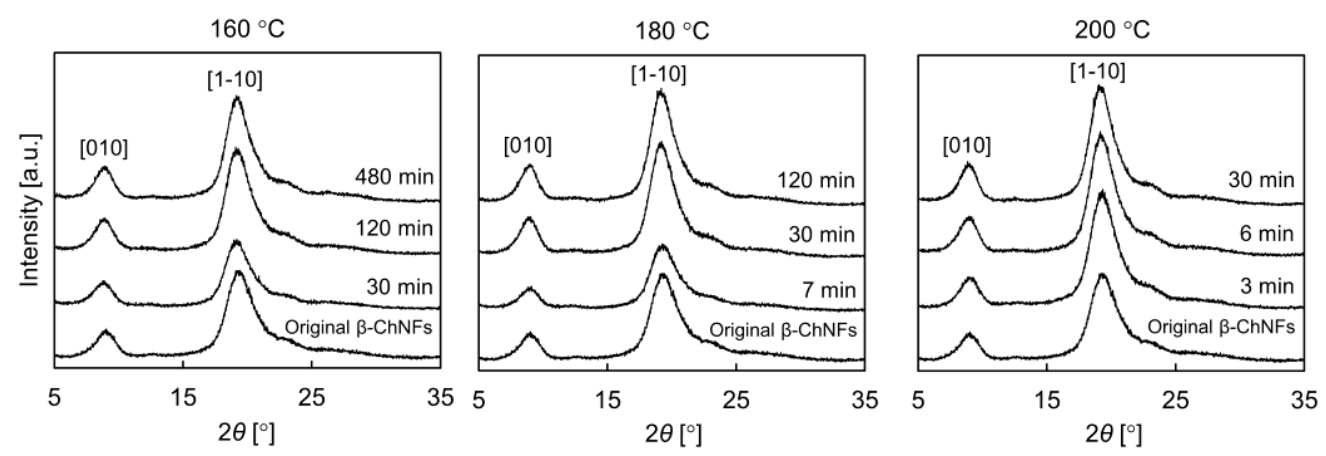

Fig. 6 XRD patterns of $\beta$-ChNFs recorded before and after the hydrothermal treatment

Table $1 \mathrm{CI}, d$-spacing, and CS values determined for $\beta$-ChNFs before and after the hydrothermal treatment

\begin{tabular}{|c|c|c|c|c|c|c|c|c|}
\hline \multirow{2}{*}{$\begin{array}{c}\text { Heating } \\
\text { temperature }\left[{ }^{\circ} \mathrm{C}\right]\end{array}$} & \multirow{2}{*}{$\begin{array}{l}\text { Heating } \\
\text { time [min] }\end{array}$} & \multirow{2}{*}{$\log R_{0}$} & \multicolumn{2}{|c|}{$\mathrm{CI}^{a}[\%]$} & \multicolumn{2}{|c|}{$d$-spacing $[\AA]$} & \multicolumn{2}{|c|}{$\mathrm{CS}^{b}[\mathrm{~nm}]$} \\
\hline & & & [010] & [1-10] & [010] & [1-10] & [010] & [1-10] \\
\hline Original $\beta$-ChNFs & & & 58 & 83 & 9.8 & 4.6 & 12.0 & 6.7 \\
\hline \multirow[t]{3}{*}{160} & 30 & 4.9 & 64 & 83 & 10.1 & 4.6 & 10.5 & 7.2 \\
\hline & 120 & 5.6 & 67 & 87 & 10.0 & 4.7 & 10.0 & 7.2 \\
\hline & 480 & 6.2 & 65 & 87 & 9.9 & 4.6 & 11.4 & 7.6 \\
\hline \multirow[t]{3}{*}{180} & 7 & 4.7 & 62 & 84 & 9.7 & 4.6 & 11.0 & 6.6 \\
\hline & 30 & 5.6 & 64 & 86 & 10.0 & 4.6 & 11.4 & 7.5 \\
\hline & 120 & 6.2 & 67 & 87 & 9.9 & 4.6 & 11.6 & 7.8 \\
\hline
\end{tabular}




\begin{tabular}{ccccccccc}
\hline 200 & 3 & 4.5 & 56 & 86 & 9.8 & 4.6 & 11.9 & 7.0 \\
& 6 & 5.2 & 62 & 86 & 9.8 & 4.6 & 10.7 & 7.5 \\
& 30 & 6.2 & 68 & 87 & 10.0 & 4.7 & 11.7 & 8.0 \\
\hline
\end{tabular}

${ }^{a} \mathrm{CI}$ : crystallinity index; ${ }^{b} \mathrm{CS}$ : crystal size.

Discussion on the severity factor and a comparison between chitin and cellulose NF hydrogel reported previously

The obtained results suggest that the process of hydrogel formation can be characterized by an Arrhenius-type equation. The $\log R_{0}$ value of 14.75 was originally used to describe the swelling, dissolution, and degradation of cell wall components during the hydrothermal treatment conducted at different temperatures (Overend and Chornet 1987). Interestingly, when this particular value was utilized for hydrothermal gelation, the physicochemical properties of the resulting hydrogel were very similar. Hence, the activation energy for the hydrogel formation of $\beta$-ChNFs prepared at $\mathrm{pH}=4$ and 30 passes can be unexpectedly close to the value obtained for the degradation of cell wall components. On the other hand, TEMPO-oxidized cellulose NFs were successfully converted to a self-sustaining hydrogel in our previous study by the hydrothermal treatment at a temperature of $160^{\circ} \mathrm{C}$ and shorter heating time as compared to that utilized for $\beta$-ChNFs (Suenaga and Osada 2018a). At the same time, the width, surface charge, and charge density of the TEMPO-oxidized cellulose NFs significantly differed from those of $\beta$-ChNFs. The physicochemical properties of a hydrogel consisting of various NFs can be evaluated by adding a parameter describing the properties of NFs to the equation of the severity factor. For this reason, NFs with various characteristics should be transformed to selfsustaining hydrogels via the hydrothermal gelation followed by the comparison of their properties.

From the results presented above, it was found that the physicochemical properties of $\beta$-ChNF hydrogels could be determined by calculating the value of $\log R_{0}$ regardless of the heating temperature and time. The width of $\beta$-ChNFs increased with increasing $\log R_{0}$, and no significant changes in their chemical and crystal structures were observed after the hydrothermal treatment at temperatures below $200{ }^{\circ} \mathrm{C}$.

\section{Hydrothermal gelation of $\alpha$ - and $\beta$-ChNFs prepared at neutral and acidic $\mathrm{pH}$ and different numbers of passes}

\section{Hydrothermal gelation of ChNF subjected to 10 passes}

The hydrothermal gelation of the $\alpha$ - and $\beta$-ChNFs prepared at $\mathrm{pH}=4$ and 7 and subjected to 10 passes was conducted at $180{ }^{\circ} \mathrm{C}$. The breaking strengths and compressive moduli of the resulting specimens are shown in Fig. 7, and the photographs of the hydrogels produced under these conditions are displayed in Figs. S6 and S7. As shown in Fig. S6, the $\alpha-$ ChNFs prepared at $\mathrm{pH}=7$ and 10 passes flowed after the hydrothermal treatment, and their appearances were almost identical to those of the original dispersion. Thus, these specimens could not be subjected to compression testing. Moreover, after the $\alpha$-ChNFs prepared at $\mathrm{pH}=$ 4 and subjected to 10 passes were heated to $180{ }^{\circ} \mathrm{C}$ for $120 \mathrm{~min}$, they retained a cylindrical shape; however, their breaking stress was only $0.28 \mathrm{kPa}$. For comparison, the $\beta$-ChNFs subjected to 10 passes were converted to self-sustaining hydrogels by heating to $180{ }^{\circ} \mathrm{C}$ for 30 min even at the neutral $\mathrm{pH}$, but their strength remained at a level of only $0.32 \mathrm{kPa}$ after $120 \mathrm{~min}$ of heating. Hence, the $\beta$-ChNFs prepared at $\mathrm{pH}=4$ exhibited the highest mechanical strength among the ChNF samples subjected to 10 passes. It has been reported previously that $\beta$-chitin is easily disintegrated to thinner NFs as compared to those obtained from $\alpha$-chitin (Fan et al. 
2008; Suenaga et al. 2017), which form a denser network structure in dispersion. This denser network structure becomes cross-linked by the mutual attachment of individual NFs during the hydrothermal treatment, resulting in the formation of a self-sustaining hydrogel. Because $\beta$ chitin tends to disintegrate into thinner NFs at acidic $\mathrm{pH}$ due to electrostatic repulsion (Dutta et al. 2013), the strengths of the $\beta$-ChNF hydrogels prepared at $\mathrm{pH}=4$ exhibited the highest values.

\section{Hydrothermal gelation of ChNF subjected to 30 passes}

Next, the hydrothermal gelation of the $\alpha$ - and $\beta$-ChNFs prepared at $\mathrm{pH}=4$ and 7 and subjected to 30 passes was conducted at a temperature of $180{ }^{\circ} \mathrm{C}$ to test the hypothesis that thinner NFs formed hydrogels with higher strengths (Fig. 7). Similar to the original dispersion, the $\alpha$-ChNFs prepared at $\mathrm{pH}=7$ and 30 passes also flowed after the hydrothermal treatment (see Fig. S7). The mechanical strength of the $\alpha$-ChNFs fabricated at $\mathrm{pH}=4$ increased because of the larger number of disintegration passes, and after heating to $180{ }^{\circ} \mathrm{C}$ for $120 \mathrm{~min}$, the hydrogel of $\alpha$-ChNFs prepared at $\mathrm{pH}=4$ became self-sustained without swelling. While no significant differences were observed between the $\beta$-ChNFs prepared at $\mathrm{pH}=4$ and subjected to 10 and 30 passes, the mechanical strength of the $\beta$-ChNFs fabricated at $\mathrm{pH}=7$ and 30 passes was significantly higher than that of the $\beta$-ChNFs prepared at the same $\mathrm{pH}$ and 10 passes, and its value was the highest one among the ChNF specimens subjected to 30 passes. It was also found that the decrease in the NF width accompanied by fiber disintegration increased the strength of the produced hydrogel.

\section{Effect of $\mathrm{pH}$ on the hydrothermal gelation}

To examine the influence of $\mathrm{pH}$ on the hydrogel mechanical strength, after $\beta$-ChNFs were prepared at $\mathrm{pH}=7$, their $\mathrm{pH}$ value was reduced to 4 by the addition of acetic acid (these specimens are further referred to as the $\beta$-ChNFs prepared at $\mathrm{pH}=7 \rightarrow 4$ in this work). As shown in Fig. 7, the compressive modulus of the $\beta$-ChNFs prepared at $\mathrm{pH}=7 \rightarrow 4$ and subjected to 10 passes was greater than that of the $\beta$-ChNFs fabricated at $\mathrm{pH}=7$ and the same number of passes; however, the mechanical strength of the $\beta$-ChNFs prepared at $\mathrm{pH}=7 \rightarrow 4$ and subjected to 30 passes dramatically decreased. The obtained results indicate that the cationization of well disintegrated ChNFs does not promote the formation of the network structure caused by the mutual attachment of individual ChNF because it increases the forces of electrostatic repulsion. Therefore, the strengths of the $\beta$-ChNFs subjected to 30 passes and fabricated at $\mathrm{pH}=7 \rightarrow 4$ and $\mathrm{pH}=4$ were smaller than that of the $\beta$-ChNFs prepared at $\mathrm{pH}=7$ and 30 passes. Hence, it can be concluded that the thickness of NFs is the most important factor affecting the strength of the hydrogel produced by the hydrothermal gelation. As has been mentioned earlier, acid addition sometimes improves the mechanical strength of materials (such as $\alpha$-ChNFs); however, if thinner NFs are already obtained, the forces of electrostatic repulsion should be reduced. Indeed, as was shown in our previous study, the hydrothermal gelation of the thinnest TEMPO-oxidized cellulose NFs resulted in the formation of a self-sustaining hydrogel (Suenaga and Osada 2018a). During this process, the carboxylate groups representing the main source of electrostatic repulsion were eliminated from the NF surface, which promoted the mutual adherence of TEMPO-oxidized cellulose NFs. As a result, the latter exhibited a higher compressive modulus as compared to those of the ChNF samples. Thus, the $\alpha$-ChNFs prepared at $\mathrm{pH}=4$ can be potentially transformed to a hydrogel with higher mechanical strength by increasing the number of passes and heating time. 

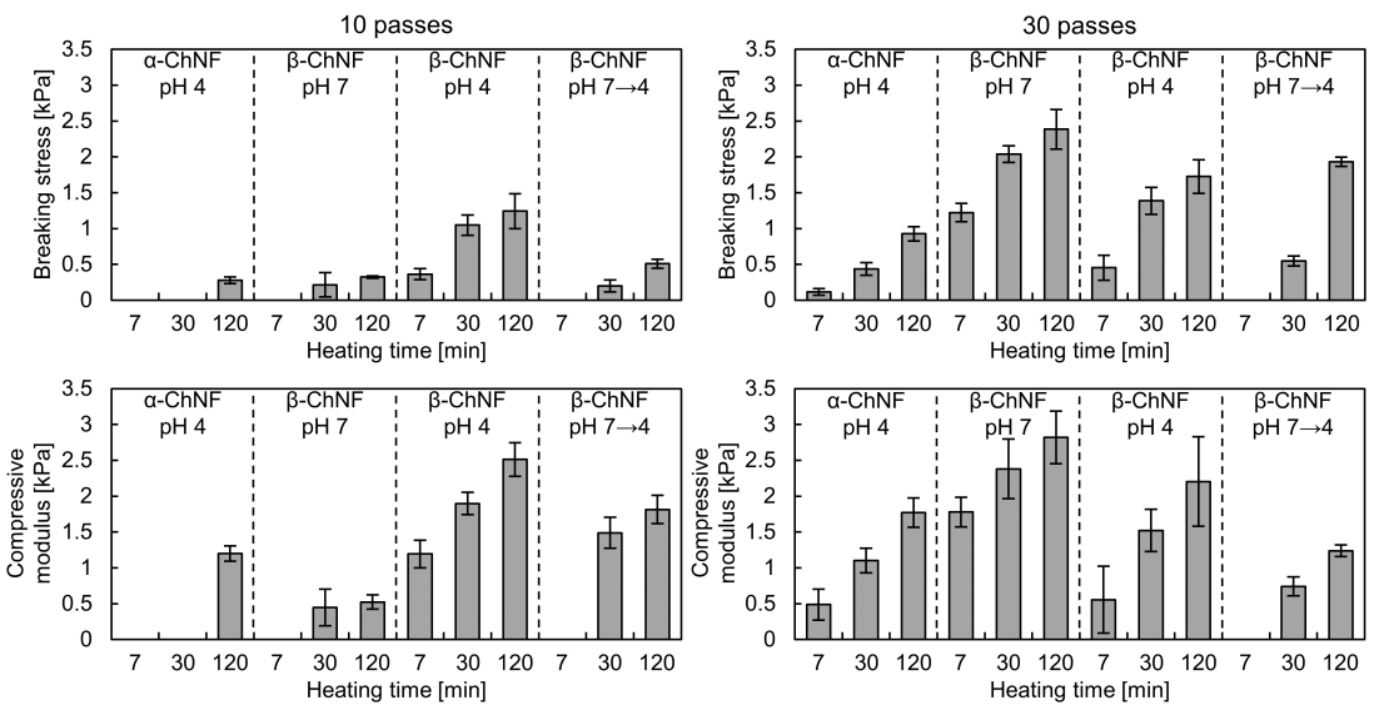

Fig. 7 Mechanical properties of the $\alpha$ - and $\beta$-ChNF hydrogels heated to $180{ }^{\circ} \mathrm{C}$

\section{Conclusions}

First, the hydrothermal treatment of the $\beta$-ChNFs prepared at $\mathrm{pH}=4$ and subjected to 30 passes was performed at $120,160,180$, and $200{ }^{\circ} \mathrm{C}$, and the severity factor $\log R_{0}$ was introduced to determine the optimal heating time. The optical transmittances and mechanical strengths of the hydrogels plotted against $\log R_{0}$ produced unique curves, suggesting that the latter parameter could be used for predicting the physicochemical properties of the hydrogels fabricated via the hydrothermal gelation. While the chemical and crystal structures of the $\beta$ ChNFs prepared at $\mathrm{pH}=4$ and 30 passes remained almost the same after the hydrothermal gelation, their width increased from $10.1 \mathrm{~nm}$ to around $15 \mathrm{~nm}$ with an increase in $\log R_{0}$, indicating the formation of a network structure via the adherence of neighboring $\beta$-ChNFs. Next, the $\alpha$ - and $\beta$-ChNFs fabricated at $\mathrm{pH}$ of 4 and 7 and subjected to 10 and 30 passes were heated to $180{ }^{\circ} \mathrm{C}$ for 7,30 , and $120 \mathrm{~min}$. After the hydrothermal treatment, the $\alpha$-ChNFs prepared at $\mathrm{pH}=7$ exhibited the same flowability as that of the original dispersion (even after 30 passes). The $\alpha$-ChNFs fabricated at $\mathrm{pH}=4$ were unable to achieve gelation after 10 passes; however, after 30 passes, their mechanical strength increased with an increase in the heating time. While the influence of the number of passes on the strength of the $\beta$-ChNFs prepared at $\mathrm{pH}=4$ was insignificant, the strength of the $\beta$-ChNFs fabricated at $\mathrm{pH}=7$ dramatically increased with increasing its magnitude from 10 to 30 and reached maximum among the studied $\mathrm{ChNF}$ specimens. The strength of the $\beta$-ChNFs fabricated at $\mathrm{pH}=7 \rightarrow 4$ was significantly smaller than that of the $\beta$-ChNFs prepared at $\mathrm{pH}=7$ and 30 passes. Hence, it can be concluded that the hydrothermal gelation can be optimized using thin ChNFs and controlling the forces of electrostatic repulsion. Furthermore, the data obtained in this study may be potentially used for expanding the scope of engineering applications of the hydrothermal gelation technique.

\section{Acknowledgements}

This work was supported by JSPS KAKENHI [grant number: 17H04893]. We thank Dr. Nobuhide Takahashi, Dr. Hiroshi Fukunaga, Dr. Iori Shimada, Dr. Kazuhide Totani, Dr. Yoshihiro Nomura, and Mr. Kazuhiko Yamashita 
for their substantial intellectual contributions to the conception of the project.

\section{Conflict of Interest}

The authors declare that they have no conflict of interest. 


\section{References}

Abe K, Ifuku S, Kawata M, Yano H (2014) Preparation of tough hydrogels based on $\beta$-chitin nanofibers via $\mathrm{NaOH}$ treatment. Cellulose 21:535-540

Aida TM, Oshima K, Abe C, Maruta R, Iguchi M, Watanabe M, Smith RL (2014) Dissolution of mechanically milled chitin in high temperature water. Carbohydr Polym 106:172-178

Brugnerotto J, Lizardi J, Goycoolea FM, Argüelles-Monal W, Desbrières J, Rinaudo M (2001) An infrared investigation in relation with chitin and chitosan characterization. Polymer 42:3569-3580

Chang C, Chen S, Zhang L (2011) Novel hydrogels prepared via direct dissolution of chitin at low temperature: structure and biocompatibility. J Mater Chem 21:3865-3871

Dutta AK, Izawa H, Morimoto M, Saimoto H, Ifuku S (2013) Simple preparation of chitin nanofibers from dry squid pen $\beta$-chitin powder by the star burst system. J Chitin Chitosan Sci 1:186-191

Fan Y, Saito T, Isogai A (2008) Preparation of chitin nanofibers from squid pen $\beta$-chitin by simple mechanical treatment under acid conditions. Biomacromolecules 9:1919-1923

Gautier S, Xhauflaire-Uhoda E, Gonry P, Piérard GE (2008) Chitin-glucan, a natural cell scaffold for skin moisturization and rejuvenation. Int J Cosmet Sci 30:459-469

Han LK, Kimura Y, Okuda H (1999) Reduction in fat storage during chitin-chitosan treatment in mice fed a highfat diet. Int J Obes 23:174-179

Izumi R, Komada S, Ochi K, Karasawa L, Osaki T, Murahata Y, Tsuka T, Imagawa T, Itoh N, Okamoto Y, Izawa H, Morimoto M, Saimoto H, Azuma K, Ifuku S (2015) Favorable effects of superficially deacetylated chitin nanofibrils on the wound healing process. Carbohydr Polym 123:461-467

Jayakumar R, Divya Rani VV, Shalumon KT, Kumar PS, Nair SV, Furuike T, Tamura H (2009) Bioactive and osteoblast cell attachment studies of novel $\alpha$ - and $\beta$-chitin membranes for tissue-engineering applications. Int $\mathbf{J}$ Biol Macromol 45:260-264

Klinchongkon K, Khuwijitjaru P, Wiboonsirikul J, Adachi S (2015) Extraction of oligosaccharides from passion fruit peel by subcritical water treatment. J Food Process Eng 40:e12269.

Lewis L, Derakhshandeh M, Hatzikiriakos SG, Hamad WY, MacLachlan MJ (2016) Hydrothermal gelation of aqueous cellulose nanocrystal suspensions. Biomacromolecules 17:2747-2754

Madhumathi K, Binulal NS, Nagahama H, Tamura H, Shalumon KT, Selvamurugan N, Nair SV, Jayakumar R (2009) Preparation and characterization of novel $\beta$-chitin-hydroxyapatite composite membranes for tissue engineering applications. Int J Biol Macromol 44:1-5

Mushi NE, Kochumalayil J, Cervin NT, Zhou Q, Berglund LA (2016) Nanostructurally controlled hydrogel based on small-diameter native chitin nanofibers: preparation, structure, and properties. ChemSusChem 9:989-995

Nata IF, Wang SSS, Wu TM, Lee CK (2012) $\beta$-Chitin nanofibrils for self-sustaining hydrogels preparation via hydrothermal treatment. Carbohydr Polym 90:1509-1514

Osada M, Kikuta K, Yoshida K, Totani K, Ogata M, Usui T (2013) Non-catalytic synthesis of chromogen I and III from $N$-acetyl-D-glucosamine in high-temperature water. Green Chem 15:2960-2966

Osada M, Miura C, Nakagawa YS, Kaihara M, Nikaido M, Totani K (2012) Effect of sub- and supercritical water pretreatment on enzymatic degradation of chitin. Carbohydr Polym 88:308-312

Osada M, Miura C, Nakagawa YS, Kaihara M, Nikaido M, Totani K (2015) Effect of sub- and supercritical water treatments on the physicochemical properties of crab shell chitin and its enzymatic degradation. Carbohydr Polym 134:718-725

Overend RP, Chornet E (1987) Fractionation of lignocellulosics by steam-aqueous pretreatments. Philos Trans R Soc A 321:523-536

Shen X, Shamshina JL, Berton P, Bandomir J, Wang H, Gurau G, Rogers RD (2016) Comparison of hydrogels prepared with ionic-liquid-isolated vs commercial chitin and cellulose. ACS Sustain Chem Eng 4:471-480

Sowmya S, Kumar PTS, Chennazhi KP, Nair SV, Tamura H, Jayakumar R (2011) Biocompatible $\beta$-chitin hydrogel/nanobioactive glass ceramic nanocomposite scaffolds for periodontal bone regeneration. Trends Biomater Artif Organs 25:1-11

Suenaga S, Nikaido N, Totani K, Kawasaki K, Ito Y, Yamashita K, Osada M (2016) Effect of purification method of $\beta$-chitin from squid pen on the properties of $\beta$-chitin nanofibers. Int J Biol Macromol 91:987-993

Suenaga S, Osada M (2018a) Self-sustaining cellulose nanofiber hydrogel produced by hydrothermal gelation without additives. ACS Biomater Sci Eng 4:1536-1545

Suenaga S, Osada M (2018b) Systematic dynamic viscoelasticity measurements for chitin nanofibers prepared with various concentrations, disintegration times, acidities, and crystalline structres. Int J Biol Macromol 115:431437

Suenaga S, Totani K, Nomura Y, Yamashita K, Shimada I, Fukunaga H, Takahashi N, Osada M (2017) Effect of acidity on the physicochemical properties of $\alpha$ - and $\beta$-chitin nanofibers. Int J Biol Macromol 102:358-366

Tamura H, Nagahama H, Tokura S (2006) Preparation of chitin hydrogel under mild conditions. Cellulose 13:357364

Wagner W, Pruß A (2002) The IAPWS formulation 1995 for the thermodynamic properties of ordinary water 
substance for general and scientific use. J Phys Chem Ref Data 31:387-535

Yan N, Chen X (2015) Don't waste seafood waste. Turning cast-off shells into nitrogen-rich chemicals would benefit economies and the environment. Nature 524:155-158

Zhang Y, Xue C, Xue Y, Gao R, Zhang X (2005) Determination of the degree of deacetylation of chitin and chitosan by X-ray powder diffraction. Carbohydr Res 340:1914-1917 
Graphical abstract

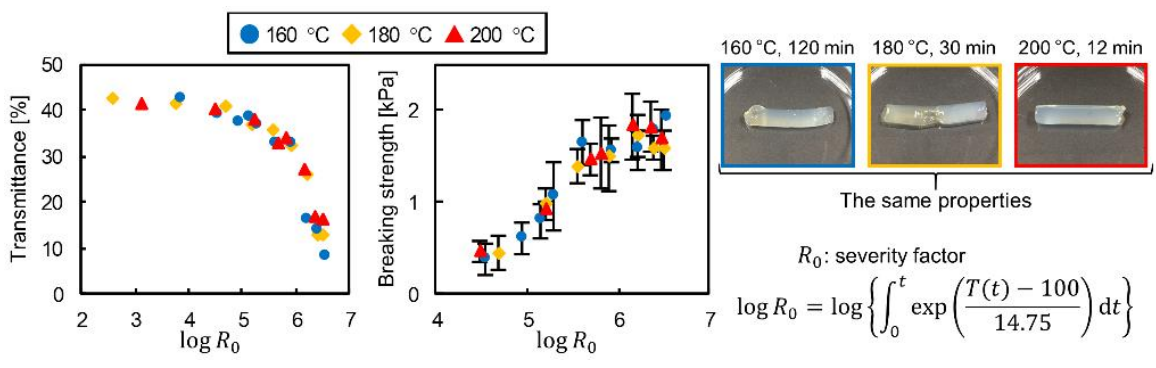

